\title{
Focus on psychiatry in Egypt
}

\section{A. OKASHA}

Mental disorders have been recognised in Egypt for millennia; 5000 years ago, they were considered to be physical ailments of the heart or uterus, as described in the Ebers and Kahun papyri (Okasha, 2001). These disorders carried no stigma, as there was no demarcation then between psyche and soma. In the 14th century -600 years before similar institutions were founded in Europe - the first psychiatric unit was established, in Kalaoon Hospital in Cairo.

Egypt is central to the Arab world, which, despite its wealth and its natural and human resources, has fared poorly in many aspects of development. Important problems include illiteracy (especially among women), lack of job opportunities (especially for young people) and slow economic growth because of loss of traditional economies, low productivity, and lack of innovation and competitiveness. Military spending is triple that of other regions. Rapid expansion of Arab populations threatens progress, especially in countries with limited resources such as Egypt.

\section{PSYCHIATRIC EDUCATION IN EGYPT}

Egypt has 17 medical schools; all of them have psychiatric departments, seven of which are departments of neuropsychiatry. They have offered a diploma of neuropsychiatry for more than 60 years, a master's degree in psychiatry for the past 25 years and a doctorate for the past 20 years. Students must complete a thesis and written, oral and clinical examinations.

\section{MENTAL HEALTH SERVICES}

Egypt, with a population of over 70 million, has about a thousand psychiatrists (one psychiatrist for approximately 70000 citizens), more than 1300 psychiatric nurses and about 200 clinical psychologists, with hundreds of general psychologists working in fields unrelated to mental health services. There are many social workers practising in all psychiatric facilities, but unfortunately they are general social workers with minimal graduate training in psychiatric social work. In 1960 there was an attempt to educate psychiatric social workers at the Institute of Social Services in Cairo, but this lasted for only 2 years because of a shortage of applicants.

Egypt has about 9700 psychiatric beds, one bed for every 7000 citizens (i.e. 15 beds per 100000 population), constituting less than $10 \%$ of the total number of hospital beds (110000). The two largest mental hospitals in Egypt were facing great difficulties regarding care, finances, treatment and rehabilitation while accommodating about 5000 patients. Three new hospitals, each providing 300 beds, have now been built on the premises of these two hospitals, with a view to providing sufficient mental health services of the highest quality.

The new policy of deinstitutionalisation and the provision of community care may reduce the number of psychiatric inpatients, but will not solve the problem. Aftercare services in Egypt are still limited, owing to the poor understanding of most people of the need for follow-up care after initial improvement. Community care in the form of hostels, day centres, rehabilitation centres and health visitors is only available in major cities; otherwise it is provided by the family (Okasha \& Karam, 1998).

\section{MENTAL HEALTH POLICY}

The National Mental Health Programmes 1991-1996 and 1997-2003 focused on the inclusion of mental health in primary health care, training family doctors to deal with the main bulk of mental disorders, and raising public awareness regarding recognition of mental disorders and referral routes. The future policy of psychiatric services in Egypt is to build medium-stay hospitals of 600 beds, which will serve three neighbouring governorates, and short-stay hospitals of 100 beds. The encouragement of intensive psychiatric out-patient treatment in all general hospitals is proposed.

Mental health legislation was introduced in Egypt in 1944, in advance of most other Arab and African countries. Most of the existing laws dealing with mental health are now old, having been written prior to the new concepts of community psychiatry and the integration of mental health into other health services (Okasha \& Karam, 1998). An attempt to update them is now in progress.

\section{PROFILE OF PSYCHIATRIC DISORDERS IN EGYPT}

\section{Hysteria (conversion or dissociative disorder)}

Hysteria occupies a position at the top of the list of psychiatric diagnoses. There has been much controversy as to the relevance of its nosological status. In 1990, the first 1000 people presenting to the out-patient clinic of the Institute of Psychiatry of Ain Shams University in Cairo were screened to determine whether they fulfilled DSMIII-R criteria for either conversion or dissociation disorder (Okasha et al, 1993a), replicating a study undertaken at an Egyptian university hospital 23 years earlier, where hysteria constituted $11.2 \%$ of the sample (Okasha, 1967). The newer study aimed to test the relevance of the diagnosis of 'hysteria' (conversion and dissociative disorder). According to its results, many disorders that would formerly have been diagnosed as hysteria would now receive another diagnosis, mostly somatoform disorder. However, some disorders still require the diagnostic label of hysteria to reflect the symptoms and the underlying mechanisms (stress, primary gain, secondary gain, and motor or sensory symptoms that are culturally and symbolically specific for the stress). The prevalence of $5 \%$ in that study is comparable with that of organic mental disorders $(5.1 \%)$, personality disorders $(4.9 \%)$ and anxiety disorders $(7.9 \%)$, indicating that hysteria cannot be ignored as a diagnostic category. Factors that might contribute to a real decline in the incidence of hysteria could be related to the industrialisation of Egyptian society and its increasing complexity, for which 
the primitive mechanism of defence against frustration is no longer strong enough to ward off anxieties. However, the decrease in the diagnosis of hysteria could also be attributed to the diagnostic system used. The ICD-10 and DSM-III-R, which do not favour the diagnosis of hysteria because of its dynamic character, contain a number of categories for which the earlier diagnosis would have been hysteria. These categories include other somatoform disorders such as somatisation, psychogenic pain disorder, hypochondriasis, body dysmorphic disorder and undifferentiated somatoform disorder not otherwise specified.

\section{Anxiety disorders}

Earlier studies of psychiatric morbidity among university students in Egypt showed that anxiety states were diagnosed in $36 \%$ of the study sample (Okasha et al, 1977). In 1981 Okasha \& Ashour undertook the first attempt to study the sociodemographic aspects of anxiety disorders in Egypt and to apply the Arabic version of the Present State Examination in evaluating the profiles of clusters and symptoms of anxiety in a sample of 120 patients with anxiety (Okasha \& Ashour, 1981). The findings revealed that the most common symptoms were worrying $(82 \%)$, irritability $(73 \%)$, free-floating anxiety $(70 \%)$, depressed mood $(65 \%)$, tiredness $(64 \%)$, restlessness $(63 \%)$, and anergia and retardation $(61 \%)$. Panic attacks were present in $30 \%$, situational anxiety in $35 \%$, specific phobias in $37 \%$ and avoidance in $53 \%$ of the sample. Male patients showed significantly more hypochondriasis and anxiety on meeting people than females. This can be explained by the fact that men in our culture tend to somatise their psychological symptoms, as the latter may lower their prestige and degrade their pride, because of the belief that 'real' men do not have psychological symptoms. Female patients showed significantly more increased free-floating anxiety, loss of weight and conversion symptoms (Okasha \& Ashour, 1981). In 1993 anxiety states represented about $22.6 \%$ of diagnoses made in a psychiatric out-patient clinic in a selective Egyptian sample (Okasha et al, 1993a).

\section{Obsessive-compulsive disorder}

A study investigating the demographic profile and symptoms of Egyptian patients with obsessive-compulsive disorder found that more than two-thirds of the patients were male. The most commonly occurring obsessions were religion and contamination $(60 \%)$ and somatic obsessions (49\%), whereas the most commonly occurring compulsions were repeating rituals $(68 \%)$, cleaning and washing compulsions $(63 \%)$ and checking compulsions (58\%). A third of patients had a comorbid depressive disorder. A comparison was drawn between the most prevalent symptoms in our sample and those of other studies performed in India, England and Jerusalem. Obsessions were found to be similar in content in Muslims and Jews, differing from those in Hindus and Christians, signifying the role of cultural and religious rituals in the presentation of obsessive-compulsive disorder. The obsessions of the patients from Egypt and Jerusalem were similar, dealing mainly with religious matters and matters related to cleanliness and dirt. Common themes between the Indian and British samples, on the other hand, were mostly related to orderliness and aggressive issues (Okasha et al, 1994).

\section{Depressive disorders}

The prevalence rates of depression among selected samples from an urban and a rural population in Egypt were found to be $11.4 \%$ and $19.7 \%$, respectively. Dysthymic disorder was the most common diagnostic category in the urban population $(4.1 \%)$, whereas adjustment disorder with depressed mood was more frequently encountered in the rural population $(6.7 \%)$. Major affective disorder according to DSM-III criteria was diagnosed in $1.9 \%$ of the urban population compared with $3.3 \%$ of the rural population; the total prevalence was $2.5 \%$ (Okasha et al, 1988).

A cross-cultural comparison between Western and Egyptian patients with depressive illness reveals some differences. Depression among Egyptian patients is manifested mainly by agitation, somatic symptoms, hypochondriasis, physiological changes such as decreased libido, anorexia and insomnia, which is not characterised by early morning awakening. Egyptian patients mask their affect with multiple somatic symptoms, which occupy the foreground, and the affective component of their illness recedes to the background. This may be because of the greater social acceptance of physical complaints than of psychological complaints, which are either not taken seriously or are believed to be cured by rest or extra praying. The increase in somatic symptoms can be explained by the seriousness with which people in a given culture view 'psychological stress' compared with physical illness. NonWestern cultures emphasise social integration rather than autonomy. When affiliation is more important than achievement, how one appears to others is vital, and shame becomes more of a driving force than guilt. In the same way, physical illness and somatic manifestations of psychological distress are more acceptable and likely to evoke a caring response than vague complaints of psychological symptoms, which can be either disregarded or considered a stigma of being 'soft' - or, even worse, insane (Okasha et al, 1977; Gawad $\&$ Arafa, 1980). Egyptians who are depressed either resort to their primary health care physician, who is likely to request unneeded and costly investigations, or ask traditional healers to alleviate their suffering. A considerable number do not ask for help at all, especially in rural populations, among which absenteeism from work or inability to face day-to-day affairs is largely tolerated by the community.

\section{Suicide and parasuicide}

Feelings of hopelessness and the intention to kill oneself are not common among Muslims, for whom losing hope in relief by God and self-inflicted death are blasphemous and punishable in the afterlife. However, rates of suicide attempts (parasuicide), which are more likely to be intended to elicit care, have no significant associations with religiousness among Arabs. Although the wish to die is not uncommon among people with depression in Arab cultures, it usually remains at the level of wishing that God would terminate their life, and does not progress to the wish to kill themselves (Fakhr el Islam, 2000).

The crude rate of suicide attempts in Cairo was found to be 38.5 per 100000 . There was a high percentage in the age group 15-44 years, with no major difference between the genders. Single patients represented $53 \%$ of the total, with students showing the highest risk (40\%). Depressive illnesses, hysterical reactions and adjustment disorders (in that order of frequency) were the main causes of the attempt. Overdose by tablet ingestion was the most commonly used method $(80 \%)$. Official government reports are misleading and do not represent the true rate; assuming that 
one in ten suicide attempts ends with actual suicide, a crude estimate of suicide in Egypt would be about 3.5 per 100000 (Okasha \& Lotaief, 1979). A study in 1981-1982 showed that the majority of suicide attempters were young women belonging to large, overcrowded families. They showed a higher tendency to be single, literate and unemployed than the corresponding age group in the general population. Drug overdose was the method most commonly used. The majority made their attempt at home when there was somebody nearby, and $31 \%$ had made previous nonserious attempts. Dysthymic disorders and adjustment, affective and personality disorders were the most common diagnoses encountered (Okasha et al, 1986).

\section{Acute psychosis}

The symptomatological and diagnostic differentiation and outcome of acute psychosis were studied in 50 Egyptian patients using the Schedule of Clinical Assessment of Acute Psychotic States (Wig \& Parhee, 1984). The prevailing symptoms were delusions, worry, irritability, mood changes and disturbed behaviour. Almost two-thirds $(64 \%)$ of the patients were symptom-free when assessed 1 year later. The category of acute and transient polymorphic psychotic disorder with or without stress in ICD-10 encompasses these clinical syndromes in different cultures (Okasha et al, 1993a).

\section{Schizophrenia}

Schizophrenia is the most common chronic psychosis in Egypt and accounts for the majority of in-patients in our mental hospitals. The nature of their delusions reflects the individual characteristics of the patients in relation to Egyptian culture. What strikes one first and foremost in schizophrenia occurring among natives of the countryside is the belief in the intervention of supernatural beings, occult forces or of magic. Persecutory delusions with ideas of reference are the rule; religious, political, scientific and sexual delusions are frequent, financial, social, health-related, emotional and autistic delusions are less common, and delusions of grandeur are uncommon. Religious delusions are frequent, owing to the highly religious nature of Egyptian society. Political delusions are positively correlated with the level of political sanctions and pressure. Sexual delusions are more common in groups in whom sexual behaviour is severely suppressed, for example single and rural patients.

Our observations revealed that catatonic forms of the disorder are relatively common compared with other varieties. The main symptoms are retardation, withdrawal, mutism and stupor, which may be interrupted by outbursts of excitement. Many patients present with an undifferentiated type of schizophrenia, exhibiting a wide variety of symptoms such as confused thinking and a turmoil of emotion manifested by perplexity, ideas of reference, fear, dream states and dissociative phenomena (Okasha, 1993b).

\section{Child and adolescent psychiatry}

Egyptian children under 5 years old $(9.5$ million) and those aged 5-16 years (14.5 million) constitute $14.8 \%$ and $24.7 \%$ of the total population, respectively. Thus, almost $40 \%$ of the Egyptian population of 67 million are under 16 years old. The number of working children under 12 years of age is more than 1 million. Egyptian children constitute $7 \%$ of the country's labour force (Central Agency for Public Mobilization and Statistics, 1992). The general public does not favour the inclusion of disorders of children and adolescents within the province of psychiatrists, although prevalence rates indicate that such disorders constitute a considerable percentage of the profile of psychiatric illness in Egypt.

\section{Emotional disorders}

In the 1999 national survey of Egyptian children and adolescents $(n=14271$, aged $10-18$ years), $59 \%$ of the sample reported experiencing feelings of fear or anxiety. Girls reported this more than boys, urban dwellers substantially more than rural dwellers $(63.2 \% v .55 .7 \%)$, adolescents of higher socio-economic status more than those of middle to lower status, and working adolescents less than non-working ones. Fear was more reported by adolescents who were in school compared with those who were not (Ibrahim et al, 1999). More than $35 \%$ of high school students showed moderate anxiety on the Taylor anxiety scale and the majority of them had a history of exposure to chronic stress. The prevalence was higher among secondary school students $(40.8 \%)$ than among preparatory school students $(32.8 \%)$, showing significant correlation with older age, neurotic traits in childhood, larger family size, lower family income, a disturbed parental relationship and parental separation by divorce rather than death (Seif El Din, 2000). Psychiatric comorbidity revealed a prevalence of $58.4 \%$, with neurotic stressrelated disorders and somatoform disorders being the most common diagnoses (Okasha et al, 1999a). Anxiety disorders were diagnosed in $7.9 \%$ and hyperkinetic disorder in $2.2 \%$ of a sample of 8459 schoolchildren aged 6-12 years. Nocturnal enuresis was present in $1.9 \%$ of children in Egyptian surveys. Bedwetting is tolerated in a child up to the age of 5-6 years; the age at which parents decide to do something about it is usually $7-10$ years. The greatest prevalence of stammering was found in two age groups: 6-7 years and 11-12 years. At all ages stammering was more prevalent in males than in females, with a gender ratio of 3.2:1 (Okasha et al, 1999b).

Another study revealed a $7.9 \%$ prevalence rate of anxiety among Egyptian primary-school children. Psychiatric comorbidity was found in $89 \%$ of the anxiety-positive sample, including mainly 'behavioural and emotional disorders with onset usually occurring in childhood' and 'neurotic, stress-related and somatoform disorders'. Forty per cent of children with anxiety disorders had a comorbid depressive disorder (Okasha et al, 1999b).

In a governorate-wide study involving a representative sample of primary and preparatory schools in the city of Alexandria $10.3 \%$ of pupils demonstrated depressive scores, which were highest among the oldest age group (20.3\%). Girls were highly represented among depression scores compared with boys. Lack of communication and presence of child-parent conflict ranked highest among predisposing factors (23.4\%), followed by parental conflicts $(20.7 \%)$ and scholastic problems $(29.8 \%)$. In $90.1 \%$ of the depressed sample there were frequent complaints of physical symptoms for more than 6 months prior to the study (Abou Nazel, 1989). Adolescents who had a positive history of suicide attempts had significantly higher depression scores $(93.7 \%$ ) (Abou Nazel et al, 1991). Egyptian children suffering from depressive episodes present to the clinic with other symptoms, such as nocturnal enuresis and headache. Ten per cent of them met conduct disorder criteria and $15 \%$ had mixed anxiety and depressive disorder according to ICD-10 criteria (Seif El Din, 1990). Frequently they receive symptomatic treatment without identification 
of the underlying psychiatric disorder (Attia et al, 1991).

\section{Behavioural disorders}

Behavioural problems in childhood are frequently interpreted as misbehaviour that can be managed by punishment or reward within the family. Within the overcrowded schools, teachers are less likely to differentiate between children with a developmental disorder, adjustment disorder or mild learning diability. Behaviour disorders represented $5 \%$ (in 1967) and $8.2 \%$ (in 1990) of diagnoses in all children attending the out-patient psychiatric facilities of the Ain Shams University hospitals (Okasha et $a l, 1993 a)$. The presenting symptoms were mainly hyperactivity, aggression, stealing and wandering. This problem was more common in patients from the cities; in Egyptian villages, conditions are conducive to the development of happy and socially secure children. Such children learn crafts and appropriate conduct smoothly from their everyday coexistence with parents and elders, and are gradually initiated into the fuller social responsibilities of the extended family community. When villagers move to the cities, their work becomes mechanised, and mothers as well as fathers work away from home. They pass on to their children little knowledge and fewer skills which could earn them the children's respect. In such circumstances, it is difficult for parents to train their children in social responsibilities, so different from those with which the parents themselves grew up; hence, delinquency and behaviour disorders tend to develop out of lack of modelling and identity crises. Since compulsory schooling is more enforced in the cities, there is also a tendency to see more cases of educational problems there.

Temper tantrums are a common complaint in families with 3-year-old children. Most of the time parents respond to the tantrum by giving their children what they want, thus aggravating this developmental problem, which constitutes $23 \%$ of behavioural problems in pre-school nurseries (Seif El Din et al, 1989). Nocturnal enuresis, particularly secondary nocturnal enuresis, is the most common type of behaviour disorder presenting to urban primary health care facilities, ranging between $63.9 \%$ and $82.5 \%$ of all behavioural disorders (Koura et al, 1993). Hyperactivity and attention-deficit symptoms are encountered significantly more often among children who are underachievers. Attention-deficit hyperactivity disorder is six times more common among boys than girls (Hassan, 1999). This disorder puts children under great pressure since they are usually treated as misbehaving both at home and at school, and most of them are exposed to corporal punishment (Youssef et al, 1998a,b). Violent behaviour was nearly 2.5 times higher among children and adolescents subjected to corporal punishment at school, and even higher among those who were subjected to this form of punishment by their caregivers. The condition is frequently associated with poor communication between adult carers and the child, leading to the use of verbal and physical punishment as a tool to control and shape the proper behaviour of the children (Youssef et al, 1998b).

Smoking and drug misuse were found to be prevalent among $6 \%$ of a large national sample. An additional $2 \%$ of children reported having tried smoking only once. Boys report smoking at considerably higher levels than girls $(11.2 \%$ v. $0.3 \%)$ and more boys than girls have tried smoking once. Working boys smoke at levels about twice as high as those of non-working boys. Peer influence was reported by $41.1 \%$ of the sample as the reason for starting smoking (Ibrahim et al, 1999).

\section{Learning difficulties}

The prevalence of scholastic underachievement in a sample of pupils at elementary schools was $42.8 \%$. Diagnoses made in this group included attention-deficit hyperactivity disorder, depression, anxiety, speech difficulties and elimination problems, none of which had been detected by their teachers. Underachievers also had significantly more physical disabilities leading to school backwardness, such as visual and hearing deficits (Hassan, 1999).

Parents are often overdemanding in relation to the academic achievement of their children, even in the earliest years, and this leads to an increase in the school drop-out rate. In an Egyptian national survey a quarter of boys aged 10-19 years and a third of girls are not at school, with the highest proportion coming from families of lower socio-economic status (Ibrahim et al, 1999). The awareness of parents and school staff of children's needs at different phases of development is often inadequate. A child who is an underachiever at school is usually labelled as 'mentally retarded' by the teachers and is referred to the student psychiatric clinic for psychological assessment. During the academic year 1998-1999 the number of children referred for this reason constituted $31.8 \%$ of the total number of referred children; after complete assessment, the percentage of children with this diagnosis dropped to $18.9 \%$ (Seif El Din, 2000).

Childhood disorders that have priority in Egyptian health planning are lifethreatening conditions such as diarrhoea and acute respiratory infections. The country has few psychiatrists specialising in childhood problems. Figures from the Central Agency for Public Mobilization and Statistics indicate that mother and child care units are gradually being replaced by urban health centres, and the same is happening to the 305 school health units, which had incorporated 17 psychiatric units across the country. The problem is not only the lack of resources for providing mental health care facilities to children, but also the attitude of the community to child mental health problems. A small percentage of general psychiatrists have an interest in child psychiatry, but their knowledge and skill are based on expertise and education acquired abroad. Egyptian universities do not offer a degree in child psychiatry in spite of the magnitude and severity of mental health problems in childhood.

\section{Drug misuse}

The 1980s witnessed a sharp rise in morbidity due to drug misuse. Since then, Egyptian community leaders at all levels have demonstrated intense concern over this problem. Estimates of the magnitude of substance addiction and the changing pattern of drug availability showed that the most commonly used drugs in the 1980s were cannabis, opium, solid and liquid hypnosedatives, heroin and finally cocaine, in descending order of frequency (Okasha, 1996). Although epidemiological data on drug misuse in Egypt are scarce, health professionals report a multitude of reasons for such concern, including an increase in the rate of addicts seeking psychiatric treatment (Al Azayem \& Ez Eldin, 1996), increases in drug-related health problems (mainly overdose toxicity; Salem, 1998) and an alarming drop in age at initiation of drug use, with a consequent rise in adolescent addicts (Amer et al, 1986). The 
average amount of heroin seized annually is about $50 \mathrm{~kg}$; this represents about $10 \%$ of total consumption. Therefore, it is safe to assume that $500 \mathrm{~kg}$ are consumed annually. If we calculate that the average daily intake is $0.25-0.5 \mathrm{~g}$ per addict, we arrive at an estimate of about 7000-10000 heroin users in Egypt (Okasha, 1996b).

During the 1990s synthetic psychoactive drug use increased exponentially to become the third most commonly available drug following cannabis and alcoholic beverages. Towards the second half of the 1990s cannabis became prevalent in the form of bango, which is prepared from leaves of Cannabis sativa. The plant is increasingly widely cultivated in Egypt, especially in the Sinai peninsula.

Epidemiological research and clinical studies of known addicts show that those who use more than one drug are double in number compared with those who use one drug exclusively. Thus, experts are rightly inclined to consider the estimate that drug seizures represent a fifth of drug use in society as more plausible. Using this formula, one can safely estimate the rate of 'experimentation' with drugs in this group to be about $10-12 \%$ in the age group 15-25 years; the rate for drug 'misuse' would be $2.5-3 \%$, whereas those identified as drug 'addicts' would constitute less than $1 \%$ of the population $(65$ million in 1998). Such estimates are alarming, and are a warning to policymakers and service providers.

The present scene in Egypt is characterised by an unprecedented shift towards 'demand reduction' at the primary prevention level, hand in hand with efforts to provide services at both secondary and tertiary health care levels. Supply control mechanisms are duly and seriously implemented (Al Akabawi, 2001).

Soueif (1994) reports different reasons for the different user categories. For secondary school students the main reason for drug use was as entertainment on happy social occasions, and the substance mostly used was hashish. Sedatives and hypnotics were the next most frequently used substances; these substances were used in situations of physical exhaustion and fatigue, and to cope with psychosocial problems or difficult working conditions, as well as at times of studying and examinations.

Egyptian surveys have found a gradual increase in the consumption of alcohol, leading to the prediction that this will be the most common form of substance misuse in the coming years. It is interesting to note that despite the relative availability of alcohol in Egypt compared with the Gulf states, the incidence of alcohol misuse is much higher in the latter countries, where the sale of alcohol is strictly prohibited on religious grounds (Okasha, 1996b).

\section{Geriatric psychiatry}

Health care systems in Egypt have largely ignored the needs of the elderly. There are only sporadic programmes to care for the elderly, mainly initiated by the community or within the private sector. Those above 65 years old represent $4.4 \%$ of Egypt's population. The country has 34 old people's homes for over a million elderly people, and some homes have waiting lists of over 1000 persons (Abyad et al, 2001).

An increasing number of elderly people live alone, or with elderly spouses and/or with only one or two other family members. The 'Care With Love' programme was established to create a sustainable, well-trained cadre of home health care providers in Egypt in order to staff units delivering such services. It was developed at the Centre for Geriatric Services in partnership with the Coptic Evangelical Organization for Social Services and As'salam Hospital in Cairo. The first training course was run in 1996, and about 500 trainees have joined the programme, taking various courses between 1996 and 2003. Ain Shams University in Cairo has started a series of courses on old age psychiatry; in addition, the Malta Institute on Ageing runs a course (in Egypt), and medical schools have started slowly to introduce lectures on ageing for undergraduates (Iskandar, 1999). Egyptian universities offer a master's degree and doctorates in geriatrics, focusing on psychogeriatrics, which is addressed as a multidisciplinary issue.

\section{THERAPIES}

\section{Psychotherapy}

Psychotherapy is an important element of psychiatric management in Egypt, with a strong religious (Muslim or Coptic) emphasis. The behaviour of individuals in Arab culture is determined more by group needs and thinking rather than by those of the individual, so that the source of control of behaviour is external rather than internal. Sources of distress and suffering on the one hand, and happiness on the other, are related to personal failure or success to achieve the expectations of significant others or of society at large. The emphasis is on conformity rather than self-actualisation. One of the objectives of psychotherapy with Arab patients is to improve adaptation by whatever means available and to focus the therapy on the manifest stress or disability. The strategy is to deal primarily with conscious problems, symptoms, thoughts, feelings and memories. Contrary to Western cultures, Arab culture is based on shame rather than guilt, so that an important motivation in social interactions is to save face and avoid being shamed. Inner desires, wishes and conflicts that are socially unacceptable must be kept secret. Inner exploration may threaten the integrity of the psyche. At the same time, gaining insight and self-realisation is socially isolating. Affects already consciously experienced by the patient should be expressed and dealt with. The therapeutic relationship should be maintained at a positive level of rapport, with deeper transference responses remaining unconscious and out of the patient's awareness. Negative transference is discussed early so that it can be dissipated as promptly as possible, allowing the patient to experience the therapist as accepting, permissive and comfortable with hostile feelings. If the patient's defences are useful and acceptable, they can be strengthened and acknowledged; if the defences are maladaptive, new ones are suggested. The therapist also tries to improve the patient's self-image by minimising the discrepancy between the patient's expectations of himself or herself (derived from the expectations of significant others) and his or her ability to realise these expectations (El Leithy, 2000).

\section{Alternative therapies}

In spite of rapid social change in Egypt, the majority of people - especially in rural areas - belong to an extended family hierarchy. It is considered shameful to care for an elderly person with dementia away from family surroundings. The parents of children with learning disabilities or hyperkinetic disorders accept primary responsibility for them, rather than having them looked after in an institution. Traditional and religious healers have a major role in primary psychiatric care in Egypt. They deal with minor neurotic, psychosomatic and transitory psychotic states using religious and group psychotherapies, 
suggestion and devices such as amulets and incantations (Okasha, 1966). About 60\% of out-patients at the university clinic in Cairo serving a population of low socioeconomic status had consulted a traditional healer before coming to the psychiatrist (Okasha et al, 1968). In rural areas of Egypt, community care is implemented without the need for health care workers. Those living in the countryside have a special tolerance of people with mental disorders and an ability to assimilate them into their community. These people, and those with mild or moderate learning disabilities, are rehabilitated daily by cultivating and planting the countryside along with, and under the supervision of, family members.

The need to add mental health care to the traditional priorities for public health care services, of bilharziasis (schistosomiasis), birth control, infectious diseases of children, smoking and illicit drug use has been gradually attracting the attention of decision-makers. Programmes for community care in big cities have been introduced in the form of out-patient psychiatric clinics, hostels for the elderly, institutions for people with learning disabilities, centres for the treatment of drug misuse, and school and university mental health services.

The National Mental Health Programme for Egypt emphasises the role of primary health care looking after $80 \%$ of psychiatric patients. Its focus is on decentralisation of mental health care and community care in different governorates. Emphasis is on recruiting mental health teams, especially psychiatric nurses, psychiatric social workers, occupational therapists and clinical psychologists.

\section{THE FUTURE}

Egypt has made substantial progress since the 1950s in reducing infant and child mortality, improving life expectancy and increasing access to health care. Major problems, however, remain. Public health challenges include high rates of maternal mortality, malnutrition, wide disparities between rural and urban areas, emphasis on curative rather than preventive care, the relative weakness of public health institutions, the variable quality of health care, lack of capacity in policy-making, and unresponsive and inequitable health systems.
The Arab Human Development report (2002) links current development status with external and internal conditions. The main external factor is military spending as a direct impediment to development, channelling resources away from development priorities such as health (including mental health). Alternative strategies conducive to development would be greater spending on technological development, empowerment of vulnerable groups, such as women and children, and promotion of democracy and human rights.

In view of the lack of human resources, mental health policies and legislation in the majority of the countries of the world, developing countries such as Egypt should develop partnerships with other agents (such as non-governmental organisations, consumer groups etc.) to provide psychiatric patients with the best care possible.

\section{REFERENCES}

Abou Nazel, M. W. (1989) Study of depression among preparatory school children. Doctoral Degree Thesis, University of Alexandria.

Abou Nazel, M., Fahmy, S., Younis, l., et al (1991) A study of depression among Alexandria preparatory school adolescents. Journal of the Egyptian Public Health Association, 66, 6 .

Abyad, A., Ashour, A. M. \& Abou-Saleh, M.T. (200I) The scope of psychogeriatrics in the Arab world. In Images in Psychiatry. An Arab Perspective (eds A. Okasha \& M. Maj), pp. 175-188. World Psychiatric Association.

Al Akabawi, A. (200I) Drug abuse in the Arab world A country profile of Egypt. In Images in Psychiatry. An Arab Perspective (eds A. Okasha \& M. Maj), pp. 143-150. World Psychiatric Association.

Al Azayem, A. \& Ez Eldin, A. G. (1996) Changing pattern of substance abuse and its reflection on management programs. Proceeding of First Egyptian International Conference on Addiction and Drug Abuse. Cairo: Ministry of Health.

Amer, H., Saleh, H., Guirguis, W., et al (1986) Use of Delphi technique in determining certain factors related to drug dependence among youth: part I. Bulletin of the High Institute of Public Health, 16, 63-77.

Arab Human Development Report (2002) Arab Human Development Report, pp. 145-149. New York: United Nations Development Program, Regional Bureau for Arab States.

Attia, M., Abou Nazel, M., Guirguis, W., et al (1991) Impact of a mental health program on the utilization of the psychiatric clinic in sporting student's hospital. Journal of the Egyptian Public Health Association, 66, 6.

Central Agency for Public Mobilization and Statistics (1992) Annual Report. Cairo: CAPMAS.

EI Leithy, W. (2000) Arab psychotherapy. In Images in Psychiatry. An Arab Perspective (eds A. Okasha \& M. Maj) pp. 247-254. World Psychiatric Association.

Fakhr el Islam (2000) Social psychiatry and the impact of religion. In Images in Psychiatry. An Arab Perspective (eds A. Okasha \& M. Maj), pp. 21-36.World Psychiatric Association.

Gawad, M. S. \& Arafa, M. (1980) Transcultural study of depressive symptomatology. Egyptian Journal of Psychiatry, 3, 163-182.

Hassan, E. (1999) Epidemiological study of scholastic underachievement among primary school children in Alexandria: prevalence and causes. Thesis, Faculty of Nursing, University of Alexandria.

Ibrahim, B., Sallam, S., El Gibaly, O., et al (1999) Transitions to Adulthood. A National Survey of Egyptian Adolescents. Cairo: Population Council.

Iskandar, M. (1999) Care With Love training programme for home health care providers. Al-Raida 16, 57-58

Koura, M., Abdel Aal, N., Khairy, A., et al (1993) Study of the role of Alexandria Primary Health Care Program in the assessment of behaviour disorders of primary school children, part II: Appraisal of school mental health services provided by primary health care facilities. Bulletin of the High Institute of Public Health, $\mathbf{2 3}$ 919-930.

Ministry of Health (1999) Health Insurance of North West of Egypt. School Health Insurance Annual Statistics Report. Alexandria: Ministry of Health.

Okasha, A. (1966) A cultural psychiatric study of El-Zar cult in UAR. British journal of Psychiatry, II2, |217-1221.

Okasha, A. (1967) Hysteria: its presentation and management in Egypt. Ains Shams Medical Journal, 18 $13-20$.

Okasha, A. (1993) Psychiatry in Egypt. Psychiatric Bulletin, I7, 548-55I.

Okasha, A. (1996a) Combat and management of drug abuse: means and challenges. An Egyptian perspective. In Proceedings of the First Egyptian International Conference on Addiction and Drug Abuse. Cairo: Ministry of Health

Okasha, A. (1996b) Substance use in a major public health hazard. In Proceedings of the First Egyptian International Conference on Addiction and Drug Abuse. Cairo: Ministry of Health.

Okasha, A. (200I) History of mental health in the Arab world. In Images in Psychiatry: An Arab Perspective (eds A. Okasha \& M. Maj), pp. I-20.World Psychiatric Association.

Okasha, A. \& Ashour, A. (198I) Psycho-demographic study of anxiety in Egypt: the PSE in its Arabic version. British Journal of Psychiatry, 139, 70-73.

Okasha, A. \& Karam, E. (1998) Mental health services and research in the Arab World. Acta Psychiatrica Scandinavica, 98, 406-413.

Okasha, A. \& Lotaief, F. (1979) Attempted suicide: an Egyptian investigation. Acta Psychiatrica Scand, 60 69-75.

Okasha, A. \& Okasha, T. (2000) Mental health in Cairo (Al-Qahira). International Journal of Mental Health, 28, $62-68$.

Okasha, A., Kamel, M. \& Hassan, A. H. (1968) Preliminary psychiatric observations in Egypt. British Journal of Psychiatry, II4, 949-955.

Okasha, A., Kamel, M., Sadek, A., et al (1977) Psychiatric morbidity among university students in Egypt. British Journal of Psychiatry, I3I, 149-154.

Okasha, A., Lotaief, F. \& El Mahallawy, N. (1986)

Descriptive study of attempted suicide in Cairo. Egyptian Journal of Psychiatry, 9, 53-90. 
Okasha, A., Khalil, A. H., El Fiky, M. R., et al (1988) Prevalence of depressive disorders in a sample of rural and urban Egyptian communities. Egyptian Journal of Psychiatry, 2, |67-|8|.

Okasha, A., Seif El Dawla, A. \& Asaad, T. (1993a) Presentation of hysteria in a sample of Egyptian patients - an update. Neurology, Psychiatry and Brain Research, I, I55-159.

Okasha, A., Seif El Dawla, A., Khalil, A. H., et a (1993b) Presentation of acute psychosis in an Egyptian sample: a transcultural comparison. Comprehensive Psychiatry, 34, 4-9.

Okasha, A., Khalil, A. H., Seif El Dawla, A., et a (1994) Phenomenology of obsessive-compulsive disorder: a transcultural study. Comprehensive Psychiatry, 35. $191-197$

Okasha, A., Bishry, Z., Ragheb, K., et al (1999a) Anxiety disorder in a sample of Egyptian adolescents: a psychodemographic study. Current Psychiatry, 6. 342-354.
Okasha, A., Bishry, Z., Seif El Dawla A., et al (1999b) Anxiety symptoms in an Egyptian sample: children and adolescents. Current Psychiatry, 6, 356-368.

Salem, S. (1998) Annual Statistical Report. Alexandria: Poison Center, Alexandria Main University Hospital.

Seif El Din, A. (1990) Evaluation of the training program for professionals working with school children in Alexandria. Alexandria Journal of Pediatrics, 4, 61-68.

Seif El Din, A. (2000) Child psychiatry in the Arab world. In Images in Psychiatry: An Arab Perspective (eds A. Okasha \& M. Maj), pp. 15I-166. World Psychiatric Association.

Seif El Din, A., Badawy, Y., Kader, E., et al (1989)

Behavioural screening for pre-school children in

Alexandria. Bulletin of the High Institute of Public Health, 19, 363-377.

Soueif, M. I. (1994) Extent and Patterns of Drug Use Among Students and Working Class Men in Egypt. Cairo: National Centre for Social and Criminological Research.
Wig, N. \& Parhee, R. (1984) Classification of acute psychosis states. Proceedings of the WHO/Asian Forum on the Status of Diagnosis and Classification of Mental Disorders and Alcohol and Drug Related Problems in the Third World.

Youssef, R., Attia, M. \& Kamel, M. (1998a) Children experiencing violence. I: Paternal use of corporal punishment. Child Abuse and Neglect, 22, 959-973.

Youssef, R., Attia, M. \& Kamel, M. (1998b) Children experiencing violence. II: Prevalence and determinants of corporal punishment in schools. Child Abuse and Neglect, 22, 975-985.

A. Okasha Director, World Health Organization Coordinating Center for Research and Training, Institute of Psychiatry, Ain Shams University, Cairo, Egypt 